\title{
PREVISÃO DAS PROPRIEDADES FÍSICAS DO PAPEL KRAFT POR ESPECTROSCOPIA NO INFRAVERMELHO PRÓXIMO (NIR) E REGRESSÃO POR MÍNIMOS QUADRADOS PARCIAIS (PLS)
}

\author{
Gisely Samistraro* e Graciela I. B. de Muniz \\ Lothário Meissner, 632, 80210-170 Curitiba - PR, Brasil \\ Patricio Peralta-Zamora e Gilcélia A. Cordeiro \\ Departamento de Química, Universidade Federal do Paraná, 81531-980 Curitiba - PR, Brasil
}

Departamento de Engenharia Florestal - Tecnologia e Utilização de Produtos Florestais, Universidade Federal do Paraná, Rua

Recebido em 25/5/08; aceito em 26/1/09; publicado na web em 3/7/09

\begin{abstract}
ESTIMATION OF PHYSICAL PROPERTIES OF KRAFT PAPER BY NEAR INFRARED SPECTROSCOPY AN PARTIAL LEAST SQUARES REGRESSION. The main objective of the present work is represented by the characterization of the physical properties of industrial kraft paper (i.e. transversal and longitudinal tear resistance, transversal traction resistance, bursting or crack resistance, longitudinal and transversal compression resistance (SCT (Compressive Strength Tester) and compression resistance (RCT-Ring Crush Test)) by near infrared spectroscopy associated to partial least squares regression. Several multivariate models were developed, many of them with high prevision capacity. In general, low prevision errors were observed and regression coefficients that are comparable with those provided by conventional standard methodologies.
\end{abstract}

Keywords: kraft paper; multivariate calibration; near infrared spectroscopy.

\section{INTRODUÇÃO}

No Brasil, a madeira para celulose e papel provém de várias espécies arbóreas de eucalipto e pinus, destacando-se Eucalyptus grandis, Eucalyptus saligna, Eucalyptus urophyla, outras em menor escala. As de pinus correspondem a Pinus elliottii, Pinus taeda e Pinus caribea.

O processo de obtenção de polpa de madeira antecede a produção de papel, sendo genericamente definido como polpação. O processo envolve a separação das fibras da madeira mediante a utilização de energia mecânica, térmica ou química ou a combinação das três. $\mathrm{O}$ processo químico tem como objetivo a dissolução da lignina, preservando a maior parte da celulose e hemicelulose.

A polpa kraft (palavra alemã que significa forte) origina papéis de maior resistência e coloração escura. ${ }^{1}$ De acordo com o mesmo autor, as principais variáveis que afetam o processo de polpação kraft estão relacionadas com as características dos cavacos de madeira (espécie, qualidade, umidade, densidade), do licor de cozimento (concentração de álcali ativo), do controle da polpação (relação licor:madeira, temperatura, pressão, curva tempo/temperatura (fator $\mathrm{H}$ )), além de outros parâmetros como grau de deslignificação desejado (indicado pelo número kappa) e álcali residual. De maneira geral, as empresas buscam uma variação mínina no processo e na qualidade da polpa, controlando rigorosamente as variáveis de polpação. Desta maneira, consegue-se um produto uniforme, com qualidade compatível com as especificações finais, delimitadas pelos testes de resistência do papel.

Antes da polpa ser encaminhada à máquina de papel passa por um processo chamado de refinação, em que as fibras são submetidas à ação mecânica, tendo como consequência penetração de água na parede celular, ruptura de algumas ligações de hidrogênio entre fibras e fibrilação da parede primária, o que contribui com o aumento da flexibilidade das fibras e melhora do entrelaçamento. ${ }^{1,2}$

Um aumento no processo de refinação provoca decréscimo da resistência ao rasgo, devido ao aumento do número de cortes nas fibras, e aumento da resistência ao arrebentamento e à tração, consequência do aumento da área de contato entre as fibras. Ainda provoca

\footnotetext{
*e-mail: giselysamistraro@yahoo.com.br
}

um decréscimo nas propriedades de alvura, opacidade e porosidade do papel, pois as folhas se tornam mais densas. ${ }^{3}$

Na máquina de papel, a suspensão de fibras é submetida ao processo de formação sobre a tela formadora e secagem. ${ }^{1,2}$ Após o papel pronto, diversos testes são aplicados com o intuito de avaliar a qualidade do produto final ("Qualidade implica em máximo atendimento das especificações e mínima geração de refugos por desclassificações e quebras na máquina de papel"). ${ }^{4}$

Dentre as variáveis que mais influenciam a qualidade do papel produzido estão o comprimento das fibras, a espessura da parede celular, os danos físicos durante o processo de refinação, os danos químicos durante o processo de polpação e deslignificação. ${ }^{3}$

As propriedades dos papéis são determinadas em ambiente padronizado por serem muito dependentes do conteúdo de umidade. Os ensaios podem ser de natureza mecânica (resistência à tração, ao arrebentamento, ao rasgo e à rigidez, absorção de energia de tensão (TEA), gramatura), de natureza ótica (alvura, opacidade, brilho e cor), de natureza química ( $\mathrm{pH}$, umidade), de natureza elétrica (rigidez dielétrica, condutividade), propriedades superficiais (lisura), permeabilidade a fluidos (resistência à passagem do ar). ${ }^{1-3}$

As propriedades que dependem da ligação entre fibras (tração, rasgo, arrebentamento) são melhoradas quanto maior é o entrelaçamento entre as fibras, o contato fibra-fibra e a colapsabilidade das fibras e dos lumes, esta última está associada com a compactação da folha úmida que coloca as paredes celulares mais próximas umas das outras. ${ }^{4}$

A busca por formas de tecnologia que garantam a uniformidade na qualidade dos produtos é constante na indústria de celulose e papel. Deste ponto de vista, contar com metodologias que permitam uma rápida avaliação das propriedades de relevância se apresenta extremamente importante, principalmente em função da morosidade dos métodos tradicionais de análise.

A espectroscopia no infravermelho próximo (NIR) tem se mostrado uma eficiente ferramenta no controle de qualidade de processos industriais. A literatura reporta inúmeros estudos e salienta as vantagens desta técnica, dentre as que destacam a sua natureza não destrutiva e não invasiva, a sua aplicabilidade universal (moléculas contendo ligações C-H, N-H, S-H, O-H), a sua rapidez e baixo custo, além da possibilidade 
de permitir o monitoramento on-line. Vantagens estas que a tornam interessante em diversos ramos como, por exemplo, agricultura, têxtil, alimentícia, farmacêutica, médica, polímeros e papel e celulose, esta última apresentando um avanço exponencial. ${ }^{5,6}$

Nos últimos anos, a potencialidade analítica da espectroscopia NIR foi significativamente favorecida pelo uso de ferramentas de calibração multivariada. Dentro do contexto da indústria de papel e celulose, destacam-se aplicações envolvendo determinações do número kappa, conteúdo de lignina, glucose, xilose e ácido urônico em polpa, ${ }^{7}$ assim como a avaliação de diversos parâmetros de polpação. ${ }^{8-13}$

A utilização on-line do NIR foi proposta para medir a densidade básica e teor de umidade em cavacos. ${ }^{14}$ Os modelos foram desenvolvidos separadamente, obtendo-se coeficientes de correlação de 0,85 para densidade básica e de 0,95 para teor de umidade, o que foi considerado satisfatório. Modelos utilizando infravermelho próximo para estimar lignina total, extrativos em etanol/tolueno e densidade básica em amostras de eucalipto foram também desenvolvidos, demonstrando uma elevada eficiência de previsão, além de significativa redução no tempo e custo no controle de qualidade da madeira destinada à produção de papel e celulose. ${ }^{15}$

Índices de tração, de arrebentamento e de rasgo em folhas de laboratório, dentre outras propriedades, foram avaliados utilizando espectroscopia no infravermelho próximo. ${ }^{16} \mathrm{Na}$ etapa de calibração, as correlações obtidas foram de 0,79 para tração, 0,70 para arrebentamento e 0,76 para o rasgo. Entretanto, a correlação verificada na etapa de validação externa foi considerada inadequada, principalmente em razão das modificações introduzidas pelas características do processo produtivo.

Neste trabalho foi avaliada a potencialidade da espectroscopia no infravermelho próximo, associada à regressão por mínimos quadrados parciais, em relação à determinação de propriedades físico-mecânicas do papel industrial kraft. As propriedades avaliadas foram: resistências ao arrebentamento ou estouro, ao rasgo transversal e longitudinal, à tração transversal, à compressão SCT (Compressive Strength Tester) longitudinal e transversal e RCT (Ring Crush Test).

\section{PARTE EXPERIMENTAL}

O papel industrial utilizado para a aquisição dos espectros é proveniente de polpação kraft. A gramatura compreendeu a faixa de 115 a $440 \mathrm{~g} \mathrm{~m}^{-2}$, abrangendo variações decorrentes do processo de produção de papel. O papel foi produzido em uma fábrica papeleira do norte catarinense, sendo que os testes físicos foram feitos no Laboratório da mesma fábrica.

Os valores de referência para desenvolvimento dos modelos foram obtidos pelos métodos convencionais de determinação das propriedades físicas e mecânicas do papel seguidos pela indústria de papel e celulose, conforme normas TAPPI (Technical Association of the Pulp and Paper Industry).

As propriedades avaliadas foram: resistência ao arrebentamento ou estouro: o equipamento mais utilizado para determinação desse ensaio é o aparelho Müllen. Consiste em medir a pressão hidráulica aplicada necessária para produzir o estouro do material. Este procedimento seguiu as recomendações da Norma TAPPI T 807 om 99 - "Papel - Determinação da resistência ao arrebentamento"; 19

resistência ao rasgo: o equipamento utilizado é um aparelho tipo pêndulo Elmendorf. Esse ensaio é a medida do trabalho necessário para o rasgamento completo do papel, que pode ser no sentido transversal ou longitudinal. Este procedimento seguiu as recomendações da Norma TAPPI T 141 om 98- "Papel - Determinação da resistência ao rasgo"; 19 resistência à tração: o equipamento utilizado é o dinamômetro. Para a realização desse teste, submete-se o corpo de prova a um esforço de tração uniformemente crescente até sua ruptura. Este procedimento seguiu as recomendações da Norma TAPPI T 494 om 01 - "Papel Determinação das propriedades de tração", 19

resistência à compressão: é medida como a força de compressão aplicada perpendicularmente ao corpo de prova. Este procedimento seguiu as recomendações da Norma TAPPI T 811 om 02 - "Papel Resistência à compressão de coluna" e da Norma TAPPI T 822 om 02 - "Ring Crush of Paperboard". ${ }^{19}$

Os espectros foram obtidos, a temperatura ambiente $\left(25 \pm 3{ }^{\circ} \mathrm{C}\right)$, em espectrofotômetro FemWin 900 da Femto, que opera em modo de refletância difusa. Amostras de $5 \times 5 \times 1 \mathrm{~cm}$ foram diretamente colocadas no porta amostras e analisadas na faixa espectral compreendida entre 1100 e $2500 \mathrm{~nm}$, acumulando 64 varreduras. As amostras foram armazenadas em dessecador, apresentando uma umidade média da ordem de $15 \%$.

Os modelos foram desenvolvidos a partir dos espectros de 108 amostras, enquanto que outros 30 foram reservados para validação. Para elaboração dos modelos foi utilizado o software Unscrambler versão 9.1, utilizando-se uma rotina de PLS (Partial Least Squares).

Diversos pré-processamentos de sinal foram avaliados, destacando-se primeira e segunda derivada e correção multiplicativa de sinal (MSC).

\section{RESULTADOS E DISCUSSÃO}

O espectro médio e o intervalo definido pelo desvio padrão das amostras pode ser observado na Figura 1. Esta figura evidencia duas características relevantes, uma que demonstra homogeneidade do perfil espectral (inexistência de sinais diferenciados), e outra que permite apreciar a elevada variância registrada na intensidade dos sinais monitorados. Bandas características de materiais lignocelulósicos podem ser observadas no espectro, sinais estes que têm sido utilizados na determinação de celulose em polpas de misturas de coníferas e folhosas ${ }^{10}(2270 \mathrm{~nm})$, hemicelulose em Eucalyptus camaldulensi ${ }^{17}$ (2276 e $2328 \mathrm{~nm})$, número kappa em polpas $\mathrm{kraft}^{7}(1680 \mathrm{~nm})$, lignina em polpas de mistura de coníferas e folhosas ${ }^{10}(1680 \mathrm{~nm})$ e lignina em Eucalyptus camaldulensis ${ }^{17}(1676 \mathrm{~nm})$

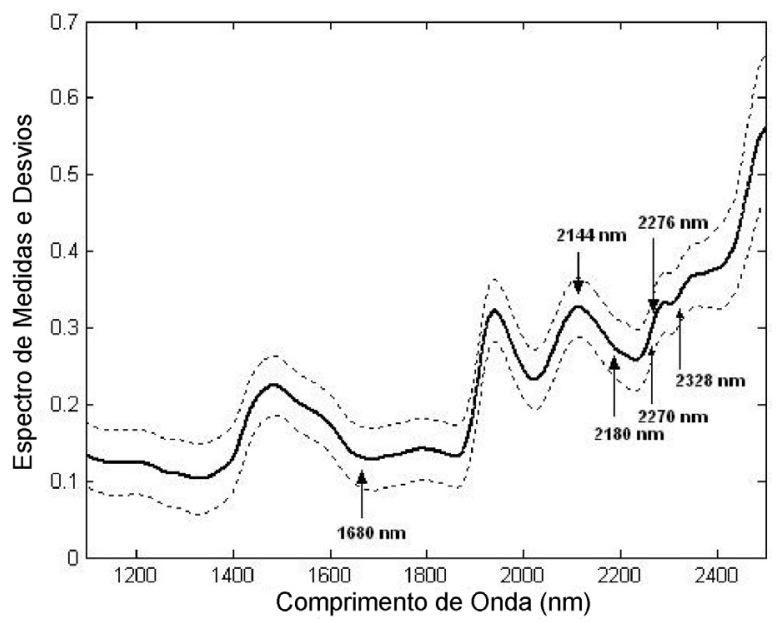

Figura 1. Espectro médio e desvio apresentado pelas amostras utilizadas na fase de calibração $(n=108)$

Na fase de desenvolvimento dos modelos de calibração, vários sistemas de pré-processamento de sinais foram ensaiados, para cada uma das propriedades avaliadas. De maneira simultânea, modelos foram desenvolvidos com vários números de variáveis latentes, sempre na procura da condição que permitisse o menor erro de previsão. Como critério de avaliação foi utilizado o valor de RMSEC, 
que corresponde à raiz quadrada da média dos erros de calibração ao quadrado, parâmetro calculado de acordo com a Equação 1.

RMSEC $=\frac{\sqrt{ } \Sigma\left(y^{\prime}-y\right)^{2}}{n}$

onde: y'é o valor conhecido, y é o valor calculado e n é o número de amostras.

Tomando como exemplo o desenvolvimento de modelos multivariados para a determinação do parâmetro "estouro", foi possível observar que o menor valor de RMSEC é viabilizado pelo uso de 9 variáveis latentes (VLs) e pré-processamento fundamentado em segunda derivada e alisamento, esta última realizada pelo ajuste polinomial móvel de Savitzki-Golay, utilizando-se janela de 15 pontos e polinômio de segunda ordem (Figura 2).



Figura 2. Efeito do número de variáveis latentes e do tipo de pré-processamento no erro de previsão na fase de calibração (RMSEC) da resistência ao estouro. Pré-Processamentos: 1: Dados centrados na média. 2: Alisamento/1 ${ }^{a}$ Derivada, 3: Alisamento/2 ${ }^{a}$ Derivada/Alisado(15 pontos), 3: Correção multiplicativa de sinal (MSC)

Trabalhando-se nestas condições, um coeficiente de correlação de 0,89 foi observado entre valores experimentais e previstos (Figura 3A), assim como uma homogênea distribuição dos resíduos (Figura 3B), para as amostras do conjunto de calibração.

Para avaliar a presença de amostras anômalas (outliers) foram utilizados os critérios de desvio de Student (com probabilidade de 95\%) e leverage. Utilizando-se os valores limites de 2,5, para o primeiro, e $3 \mathrm{VL} / \mathrm{n}$ para o segundo (onde VL representa o número de variáveis latentes e n o número de amostras) foi possível identificar amostras anômalas, as quais foram retiradas do conjunto de calibração.

Utilizando-se modelos pré-processados por alisamento e segunda derivada e fundamentados em 6 a 9 variáveis latentes, foi possível desenvolver os melhores modelos de previsão para cada um dos parâmetros avaliados. Estes resultados são sumarizados na Tabela 1.

Para a propriedade de resistência ao estouro, que está diretamente relacionada à refinação, aditivos, variações na gramatura e espessura, ${ }^{2}$ foram desenvolvidos modelos com 9 VLs. Na fase de validação, envolvendo a previsão de 30 amostras que não fizeram parte do conjunto de calibração, erros médios da ordem de 9,6 psi (RMSEV) foram observados em relação aos resultados fornecidos pelo procedimento padrão, o que representa um erro relativo médio de $1,5 \%$. O erro médio foi calculado de acordo com Equação 2 e


Figura 3. Gráfico de valores experimentais e previstos pelo modelo multivariado $(A)$ e de desvios em função do valor experimental $(B)$ na determinação de resistência ao estouro para as amostras do conjunto de calibração $(n=108)$

Tabela 1. Principais resultados obtidos nas fases de calibração $(n=108)$ e validação externa $(n=30)$, para cada uma das propriedades avaliadas

\begin{tabular}{lcccc}
\hline Propriedade & Intervalo $^{1}$ & VLs & $\begin{array}{c}\mathrm{R}^{2} \\
(\text { Fase de } \\
\text { calibração) }\end{array}$ & $\begin{array}{c}\text { Erro médio (\%) } \\
\text { (Fase de validação } \\
\text { externa) }\end{array}$ \\
\hline $\begin{array}{l}\text { Estouro } \\
\begin{array}{l}\text { Tração } \\
\text { transversal }\end{array}\end{array}$ & $22,45-171,00$ & 9 & 0,89 & 1,5 \\
$\begin{array}{l}\text { Rasgo } \\
\text { transversal }\end{array}$ & $128,0-1064,0$ & 7 & 0,92 & 3,2 \\
$\begin{array}{l}\text { Rasgo } \\
\text { longitudinal }\end{array}$ & $119,0-926,0$ & 6 & 0,91 & 8,2 \\
$\begin{array}{l}\text { SCT } \\
\text { longitudinal }\end{array}$ & $3,82-10,28$ & 6 & 0,80 & 15,1 \\
$\begin{array}{l}\text { SCT } \\
\text { transversal }\end{array}$ & $2,44-6,24$ & 8 & 0,93 & 3,0 \\
RCT & $12,6-86,0$ & 6 & 0,94 & 3,6 \\
\hline
\end{tabular}

1: Valores mínimo e máximo registrados nas amostras analisadas.

fornece a relação percentual entre os valores experimentais $(X)$ e previstos $\left(\mathrm{X}_{\mathrm{i}}\right)$, para as amostras da fase de validação. Os valores experimentais e previstos para amostras desta fase de validação são apresentados na Figura 4. 




Figura 4. Gráfico de valores experimentais e previstos pelo modelo multivariado na determinação de resistência ao estouro para as amostras do conjunto de validação $(n=30)$

$E(\%)=\left(\frac{X_{i}-X}{X}\right) \times 100$

Para o teste de tração transversal, parâmetro influenciado pelo comprimento das fibras, a formação da folha e o grau de refinação, ${ }^{2-}$ 18 o modelo foi desenvolvido com 6 VLs. Nestas condições, uma amostra se comportou como outlier, sendo eliminada do conjunto de calibração. O modelo permitiu a previsão do parâmetro com boa aproximação, com erros médios de $1,7 \mathrm{kgf}(3,2 \%)$.

A resistência ao rasgo transversal é uma propriedade influenciada por diversos fatores, dentre os quais se destacam comprimento, espessura, rigidez e orientação das fibras, assim como grau de refinação e umidade. ${ }^{2-18}$ Após retirada de três amostras consideradas anômalas, os modelos permitiram erros médios de validação de 76,8 gf, o que corresponde a erros relativos de $8,2 \%$. Na avaliação do rasgo longitudinal, quatro amostras se comportaram como outliers, sendo eliminadas da fase de calibração. Nestas condições, erros médios de $72,4 \mathrm{kgf}$ foram observados $(15,1 \%)$.

$\mathrm{Na}$ avaliação da resistência á compressão longitudinal e transversal (SCT) três amostras se comportaram como outliers. Os erros médios de previsão foram de 0,62 e $0,39 \mathrm{Kn} / \mathrm{m}$, o que representa erros relativos de 0,15 e $3,0 \%$, respectivamente.

Finalmente, na avaliação da propriedade de resistência à compressão (RCT ) sete amostras foram retiradas do modelo de calibração por se comportarem como outliers. O erro médio de previsão foi de 4,0 kgf, o que corresponde a erro relativo de 3,6\%.

De maneira geral, observa-se que todos os modelos multivariados desenvolvidos permitiram uma boa aproximação na previsão das propriedades de interesse, em relação aos valores proporcionados pelas técnicas padrão. Adicionalmente, a obtenção de coeficientes de correlação entre 0,80 e 0,94 pode ser considerada satisfatória, principalmente levando-se em consideração trabalhos similares, recentemente publicados na literatura especializada. ${ }^{14-16}$

A boa correlação observada entre os parâmetros de interesse e os dados espectrais não é surpresa, uma vez que as características que influenciam os parâmetros aqui avaliados são uma função das propriedades químicas apresentadas pelas fibras de celulose, grande parte das quais são evidenciadas na informação espectral proporcionada pelo NIR.
A refinação, por exemplo, é um processo que determina muitas propriedades mecânicas apresentadas pelas fibras de celulose. Promovendo o esgarçamento e a fibrilação da parede primária da fibra, o processo provoca a liberação de grupos hidroxila sobre a superfície externa da fibra e a absorção de água, mudanças estruturais identificadas na espectroscopia NIR.

\section{CONCLUSÃO}

De maneira geral, os resultados demonstram a potencialidade da espectroscopia no Infravermelho Próximo (NIR) em relação à avaliação das propriedades físicas do papel industrial kraft.

Os modelos elaborados permitiram a obtenção de resultados que apresentam boa concordância com os valores proporcionados pelos procedimentos padrão, apresentando coeficientes de correlação similares aos apresentados na literatura para avaliação de propriedades similares.

\section{REFERÊNCIAS}

1. Smook, G. A.; Handbook for Pulp and Paper Technologists, Tappi Press: Atlanta, 1989.

2. D’Almeida, M. H. O.; Celulose e Papel, Tecnologia de Fabricação da Pasta Celulósica, SENAI-IPT: São Paulo, 1981.

3. Sanjuan, D. R.; Obtención de Pulpas y Propiedades de las Fibras para Papel, Guadalajara: México, 1997.

4. http://www.eucalyptus.com.br/capitulos/PT03_fibras, acessada em Março 2007.

5. Williams, P.; Norris, K.; Near-Infrared Technology in Agricultural and Food Industries. $2^{\text {nd }}$ ed., American Association of Cereal Chemistry: St. Paul, 2001.

6. Pasquini, C.; J. Braz. Chem. Soc. 2003, 14, 198.

7. Olsson, R. J. O.; Tomani, P.; Karlsson, M.; Joseffson, T.; Sjoberg, K.; Bjorklund, C.; Tappi J. 1995, 78, 158.

8. Wright, J. A.; Birkett, M. D.; Gambino, M. J. T.; Tappi J. 1990, 73, 209.

9. Birkett, D. M.; Gambino, M. J. T.; Tappi J. 1989, 72, 193.

10. Easty, D. B.; Berben, S. A.; Dethomas, F.; Brimmer, P. J.; Tappi J. 1990, 73, 257.

11. Schimleck, L. R.; Michell, A. L.; Tappi J. 1999, 81, 229.

12. Antii, H.; Alexanderson, D.; Sjostrom, M.; Wallbacks, L.; Tappi J. 2000, 83, 102.

13. Skoglund, A.; Kessler, W.; Kessler, R. W.; Brundin, A.; Mandenius, C. F.; Chemom. Intell. Lab. Syst. 2004, 70, 129.

14. Caldeira, A. F.; Rocha, A. P. da; Santos, C. V. C.; Almeida. C.; Patelli, J. E.; Calvosa, P. S. P.; Sacon, V.; Resumos do $39^{\text {th }}$ Congresso Anual da Associação Brasileira Técnica de Celulose e Papel, São Paulo, Brasil, 2006.

15. Pasquini, C.; Carneiro, C. J. G.; Bomfim, P. M.; Resumos do $39^{\text {th }}$ Congresso Anual da Associação Brasileira Técnica de Celulose e Papel, São Paulo, Brasil, 2006.

16. Nisgoski, S.; Tese de Doutorado. Universidade Federal do Paraná, Brasil, 2005.

17. Terdwongworakul, A.; Punsuwan, V.; Thanapase, W.; Tsuchikawa, S.; J. Wood Sci. 2005, 51, 167.

18. Garcia, T. E.; Evaluación de la Calidad en Pulpa, Papel y Cartón. El concepto de Calidad Total, Guadalajara: México, 2002.

19. TAPPI; Test Methods 2002-2003, Tappi Press: Atlanta, 2002. 